\title{
Efficient tandem solar cells with solution-processed perovskite on textured crystalline silicon
}

Yi Hou, ${ }^{1 \dagger}$ Erkan Aydin, ${ }^{2 \dagger}$ Michele De Bastiani, ${ }^{2 \dagger}$ Chuanxiao Xiao, ${ }^{3 \dagger}$ Furkan H. Isikgor, ${ }^{2}$ DingJiang Xue, ${ }^{1}$ Bin Chen, ${ }^{1}$ Hao Chen, ${ }^{1}$ Behzad Bahrami, ${ }^{4}$ Ashraful H. Chowdhury, ${ }^{4}$ Andrew Johnston ${ }^{1}$, Se-Woong Baek, ${ }^{1}$ Ziru Huang, ${ }^{1}$ Mingyang Wei, ${ }^{1}$ Yitong Dong, ${ }^{1}$ Joel Troughton, ${ }^{2}$ Rawan Jalmood, ${ }^{2}$ Alessandro J. Mirabelli, ${ }^{2}$ Thomas Allen, ${ }^{2}$ Emmanuel Van Kerschaver, ${ }^{2}$ Makhsud I. Saidaminov, ${ }^{1}$ Derya Baran, ${ }^{2}$ Qiquan Qiao, ${ }^{4}$ Kai Zhu, ${ }^{3}$ Stefaan De Wolf, ${ }^{2 *}$ and Edward H. Sargent ${ }^{1 *}$

${ }^{1}$ Department of Electrical and Computer Engineering, University of Toronto, Toronto, Ontario M5S 1A4, Canada

${ }^{2}$ KAUST Solar Center (KSC), Physical Sciences and Engineering Division (PSE), King Abdullah University of Science and Technology (KAUST), Thuwal 23955-6900, Kingdom of Saudi Arabia

${ }^{3}$ National Renewable Energy Laboratory (NREL), Golden, Colorado 80401, USA

${ }^{4}$ Department of Electrical Engineering, Center for Advanced Photovoltaics, South Dakota State University, Brookings, South Dakota 57007, USA

$\dagger$ These authors contributed equally to this work.

*Correspondence to: E-mail: stefaan.dewolf@kaust.edu.sa (S.D.W.); ted.sargent@utoronto.ca (E.H.S.)

\begin{abstract}
Stacking solar cells with decreasing band gaps to form tandems offers to overcome the single-junction Shockley-Queisser limit in photovoltaics. The rapid development of solutionprocessed perovskites has brought perovskite single-junction efficiencies $>20 \%$. However, this process has yet to enable monolithic integration with industry-relevant textured crystalline silicon solar cells. We report tandems that combine solution-processed micrometer-thick perovskite top cells with fully-textured silicon heterojunction bottom cells. To overcome the charge-collection challenges in micrometer-thick perovskites, we enhance threefold the depletion width at the bases of silicon pyramids. Moreover, by anchoring a self-limiting passivant (1-butanethiol) on the perovskite surfaces, we enhanced the diffusion length and further suppressed phase segregation. These combined enhancements enabled independently certified power conversion efficiency of $25.7 \%$ for perovskite/silicon tandem solar cells. These devices exhibited negligible performance loss after a 400-hour thermal stability test at 85 Celsius and also after 400 hours under maximum power point tracking at 40 Celsius.
\end{abstract}


Through an intensive worldwide effort, perovskite solar cell (PSC) power conversion efficiencies (PCEs) have increased from an initial $3.8 \%$ to a remarkable certified $25.2 \%$ during the last decade $(1,2)$. This progress is based on the combination of remarkable materials properties such as a low energy required for crystal formation (3), a sharp optical absorption edge (4), and a tunable bandgap (5) ideally suited for photovoltaic (PV) applications. The rapid development of PSCs is further enabled by the use of solution-based coating methods to deposit the semiconductors (6).

These properties also make PSCs attractive as top cells for tandem applications that use lower band gap bottom cells such as crystalline silicon (c-Si) and CIGS (7-18). By reducing thermalization losses, stacking PV absorbers of decreasing band gap in a multijunction device can overcome the Shockley-Queisser efficiency limit of $33.7 \%$ for single-junction solar cells. The combination with a c-Si bottom cell is of particular appeal, as single-junction c-Si based technology has come to dominate the PV market. However, as c-Si solar cell efficiencies approach their practical limits, multijunction technologies using a c-Si bottom cell are of interest to drive further efficiency improvements.

Silicon heterojunction (SHJ) technology is attractive for tandem solar cells because of its high PCE and comparatively straightforward tandem integration. At present, most reported monolithic perovskite/silicon tandem devices are based on a single-side texturing configuration: c-Si wafers with their front flat-polished so that it is compatible with existing solution-based perovskite fabrication processes, and a textured back side for enhanced light trapping relative to the case of a double-side polished c-Si device $(8,14-16,18)$. This configuration, however, provides limited light-trapping benefits, and requires additional antireflection foils that do not provide sufficient light trapping compared to the textured counterpart (18). Further, the effectiveness of antireflection foils can be compromised upon encapsulation, and surface-polishing methods increase manufacturing cost.

As a result, recent attention has shifted to combining perovskite with fully textured c-Si (7). The benefits of fully-textured tandems have been demonstrated previously by Sahli et al. using a hybrid two-step deposition method combining sequential co-evaporation of $\mathrm{PbI}_{2}$ and $\mathrm{CsBr}$ and solution conversion (7). Unfortunately, the fill factor (FF) was moderate, a result of limited perovskite quality achieved using this approach $(19,20)$. More efficient-but also more complex - thermal co-evaporation enables textured tandems; however, the large vapor-pressure difference between the organic and inorganic components demands a high level of control over the deposition rates of each precursor during the evaporation process (21).

Early efforts to deposit perovskites by using solution techniques on top of micrometer-sized $\mathrm{Si}$ pyramids quickly revealed a number of hurdles: uncovered Si pyramids, shunt paths, and inefficient charge collection in films with variable thickness. In addition, the application of conventional surface-passivation techniques - a prerequisite for state-of-art PSC device performance - are incompatible with the rough perovskite surfaces that result from the underlying c-Si texture (7). So far, insulating solution-processed passivants have failed to cover rough surfaces with the needed consistency of thickness of a few nanometers.

Previous reports of perovskite-silicon tandems atop textured c-Si bottom cells have relied on physical vapor deposition of the perovskite front cell, rather than solution-processed perovskite 
cells on textured c-Si bottom cells. We sought to develop a high-quality micrometer-thick perovskite to cover the pyramids; and, simultaneously, to enhance charge collection in these thick films through improved drift and diffusion of photo-generated carriers. We combined solutionprocessed, micrometer-thick, wide-band gap perovskite solar cells with pyramidal-textured c-Si bottom cells. This approach achieved a threefold enhanced depletion width in the perovskite semiconductor at the valleys of Si pyramids, improving carrier collection, as revealed using nanometer-scale Kelvin probe force microscopy (KPFM).

To further increase carrier diffusion length, we introduce a conformal surface-passivation strategy for rough surfaces by anchoring a self-limiting passivant on the wide-bandgap perovskite surface. This passivant also suppresses phase segregation. In addition, micrometer-thick perovskites allow us to maintain a plateau of external quantum efficiency (EQE) of 92-93\% across the spectral range from 650 to $730 \mathrm{~nm}$. The fully-textured bottom cells minimized reflection losses and efficient light trapping was achieved for the bottom cells, crucial to satisfying current-matching conditions. Overall, with the combined enhancements in charge drift and diffusion, the best tandem cells herein achieve an independently-certified efficiency of $25.7 \%$, combined with negligible performance loss after 400 -hour thermal stability at $85^{\circ} \mathrm{C}$ and also following 400 hours under maximum power point (MPP) tracking at $40^{\circ} \mathrm{C}$.

As seen in the scanning electron microscope (SEM) top-view images, the textured c-Si features 2$\mu \mathrm{m}$-sized (111) faceted pyramids that were fabricated by alkaline wet-chemical etching (Fig. 1A). When forming tandems atop textured c-Si bottom-cell, it is important that one control the perovskite morphology and film thickness. When the perovskite film was deposited under fabrication conditions used for conventional planar perovskites (generally resulting in 400 to 600nm-thick films), pyramids that were not capped with perovskite were observed, and these regions created shunt paths in devices (Fig. S1). To cover fully the micrometer-sized pyramids, we used a concentrated $(1.65$ to $1.75 \mathrm{M})$ precursor that resulted in micrometer-thick perovskite with large (2 to $4 \mu \mathrm{m}$ ) grain sizes (Fig. 1B). This process enabled us to achieve uniform perovskite coverage of the pyramids and eliminated the need for additional flattening processes (Fig. 1, C and D).

To achieve current matching, we opted to broaden the band gap of the micrometer-thick perovskite rather than reducing the thickness of a smaller-band gap perovskite because the latter approach would uncover pyramids. This thick, wide-band gap perovskite $(1.68 \mathrm{eV}$, $\left.\mathrm{Cs}_{0.05} \mathrm{MA}_{0.15} \mathrm{FA}_{0.8} \mathrm{PbI}_{2.25} \mathrm{Br}_{0.75}\right)$ top cell also provided a path toward a higher ultimate efficiency limit in tandem solar cells (Fig. S2).

Because the size of Si pyramids and perovskite thickness were similar, this infiltrated morphology differs from previously-reported flat and conformal architectures used in perovskite-silicon tandem devices (Fig. S3) $(7,8,14,15)$. These images also suggest that the textured structures substantially modified the surface geometry by increasing the contact area. Rather than producing a conformal coating with uniform thickness, which would be similar to the evaporation case, the solution-processed perovskite smoothly overcoated the pyramid geometry while retaining the curvature of the textured surface beneath (Fig. 1D). The elemental distribution within the tandems measured via energy-dispersive x-ray spectroscopy (EDS; Fig. 1E) confirmed the presence of a textured layer stack. From the detailed features of the perovskite and conformal $\mathrm{NiO}_{\mathrm{x}}$ interface, 
we did not see undesired accumulation of hole transporter layer (HTL) material at the bottom, nor do we observe HTL material absence at the facets of the Si pyramids $(7,8,14,15)$.

Thick perovskite layers require sufficiently long charge carrier diffusion lengths to enable efficient charge collection. This condition demands perovskite crystals and surfaces of high electronic quality. To increase the performance of single-junction wide-band gap PSCs, much research has focused on developing surface treatments (22). Trioctylphosphine oxide (TOPO) can substantially reduce nonradiative recombination and increase perovskite stability (23), but its insulating nature inhibits charge transport in devices. In addition, the rough surface of textured c-Si devices sets an additional challenge in the search for surface passivation. A passivant film must conformally coat and passivate the rough top perovskite surface with readily controlled thickness and functional groups without being overly sensitive to surface topography, treatment time, and perovskite thickness. Moreover, this treatment should not dissolve the perovskite nor alter its crystal structure (24).

We pursued an approach in which we exposed the rough perovskite top surface to 1-butanethiol vapor, a technique adapted in light of previous work performed on thiol-based self-assembled monolayer growth on metal surfaces. The thiol group anchored to the perovskite surface by strong coordination of the thiol on $\mathrm{Pb}^{2+}$. The thiol molecules rapidly diffused through the vapor phase to the perovskite surface, in contrast with thiol passivation of $\mathrm{PbS}$ colloidal quantum dots, where ligand exchange occurred in solution. Because of the self-limiting nature of thiol passivation, no anchoring sites were present after the initial passivation for further growth of additional layers. Thus, prolonged exposure did not damage the perovskite.

We used ultrafast transient absorption (TA) spectroscopy to investigate the phase distribution of 1.68-eV-band gap perovskite films after different treatment times. The two classes of treated films each showed a single ground-state state bleaching peak centered at $\sim 725 \mathrm{~nm}$ (Fig. S4). This result indicated that the perovskite films existed compositionally within a single phase and the bulk properties of the perovskite film could be stabilized with the thiol self-limiting passivation (SLP) treatment. We confirmed identical recombination kinetics for the film after 30-min and 20-hour SLP treatments: the data (Fig. S4) attested to the time-insensitivity of the passivation process. Similarly, there were no differences in surface-sensitive grazing-incidence wide-angle x-ray scattering (GIWAXS) measurements between the samples with different SLP treatment times. After different treatment times, the perovskite peaks maintained their intensities and positions, suggesting no change in crystal structure and orientation (Fig. S5).

After SLP treatment, we observed in time-resolved photoluminescence (Tr-PL) an increase in the carrier lifetime from 570 ns to 900 ns when we measured the samples from air/perovskite sides. This is $\sim 15 \%$ higher than that of similar films treated with TOPO (Fig. 2A) and $\sim 60 \%$ higher than in control samples. We propose that passivation using smaller-sized thiols provided increased diffusion through the vapor phase and reduced steric hindrance. When we illuminated from the glass-perovskite side, the carrier lifetime was essentially similar in these three samples (Fig. S6). Passivation was most effective on the top surface of thick perovskite, where, in the pristine case, major carrier recombination would otherwise have occurred. 
The SLP treatment also enhanced phase stability of the perovskite. After 20 min of continuous light exposure, the SLP-treated films exhibited a stable PL peak position, whereas the control sample shows a redshift in the peak position, indicative of phase segregation (Fig. 2, B and C). This result is in agreement with earlier conclusions from Belisle et al. demonstrating that charge accumulation and carrier trapping at perovskite surfaces are drivers of photoinduced halide segregation, and that efficiently passivating the perovskite surfaces suppresses phase segregation and stabilized wide-band gap perovskites (25).

A representative randomly-selected $100 \mathrm{~nm}$ by $100 \mathrm{~nm}$ area for topographic imaging that included grain boundaries (GBs) (red square, Fig. S7) was mapped for the nanoscale charge carrier diffusion length, which is related to the local charge carrier transport time (Fig. S8) and charge carrier recombination lifetime (Fig. S9). Figures 2, D to F, present nanoscale diffusion-length maps of both control sample and SLP-treated perovskite at similar regions, indicated by the blue square. The diffusion length was improved in both grain and grain boundaries for SLP perovskites compared to control samples. The diffusion length in SLP perovskites within grains was $\sim 570 \mathrm{~nm}$. At the grain boundaries, the diffusion length of the SLP perovskite decreased by $\sim 50 \mathrm{~nm}$, whereas the diffusion length in the control sample decreased by $\sim 100 \mathrm{~nm}$ (Fig. S10). Thus, passivation of the grain boundaries for the SLP perovskite increased the charge diffusion length compared to the control sample.

To probe the effects of the textured structure on the electrical field in the vertical direction, we used nanometer-scale KPFM to profile and observe the differences in electric-potential (or electric-field) distribution across the flat and textured devices. To determine the bulk profile from the surface measurement, we applied different forward-bias voltages of 1 and $1.5 \mathrm{~V}$ to the device and imaged the cross-sectional surface potential under each voltage (Figs. S11 to S20). The junction characteristic was assessed from the small current flow, or equivalent shunt resistance, under bias, which was acquired by measuring the voltage drop across the cross-sectional surface.

By taking the first derivative of the potential difference, we determined the electric field distribution relative to the metallurgical interfaces (Fig. 3, A to I). We observed two prominent junction peaks at the HTL/perovskite and perovskite/electron-transport layer (ETL) interfaces, whereas the electric field was near zero inside the perovskite layer. The perovskite/ETL junction has similar strength and depletion width for both flat and textured cells, which agreed with the perovskite/ETL interfaces being identical for these two devices. Interestingly, the electric field at the HTL/perovskite interface was enhanced when a textured substrate was employed (Figs. 3, J and $\mathrm{K}$ ).

We determined that the depletion region in the perovskite (at the HTL/perovskite interface) was much wider at the valley of Si pyramids $(\sim 380 \mathrm{~nm})$ than at their top $(\sim 100 \mathrm{~nm})$ or compared to flat perovskite cells. This result implies that where the perovskite was the thickest, the depletion width was the largest, which would be desirable for effective charge collection. At the pyramid valleys, the perovskite was more confined and was subjected to electrical fields superimposed from neighboring HTL-coated pyramids. When Si pyramids valleys were sharper, depletion regions were wider (in a linear relation: detailed values are shown in Tables S1 and S2), which further suggested that the depletion width correlates with the geometry factor. In contrast with the case of other deposition methods (such as co-evaporation), this geometry was only observed when the 
solution-processed perovskite cells smoothened out the textured silicon cell and caused this beneficial geometry-dependent electric-field distribution. This finding agrees with the simulated results (Fig. S21).

The KPFM observation accounted for device performance trends, in that charge collection was enhanced in the best pyramid devices. The stronger and wider depletion at the valley of the pyramids benefitted charge collection of charge carriers photogenerated with long-wavelength incident light. Outside of the depletion region, the carriers needed to diffuse through the perovskite absorber layer to be collected. At the top of the Si pyramids, the depletion width was not broadened. However, here the distance between hole contact and electron contact was much shorter (100 to $300 \mathrm{~nm}$ ), which was well within the typical range of efficient charge diffusion in a PSC (Figs. 3, J and K). In light of the surrounding pyramidal structure, charges generated in the textured perovskite had a shortened transport distance to both contacts compared to a flat perovskite cell.

Figure 4A sketches the cross-section of the textured tandem. To explore the role of the thick perovskite, we studied the thickness-dependent current generation theoretically. As presented in Fig. 4B, the short-circuit current density $\left(\mathrm{J}_{\mathrm{SC}}\right)$ of the perovskite cell increased with layer thickness. The red spot represents the experimental JSC value of $\sim 1.1 \mu \mathrm{m}$-thick perovskite used in this work (Fig. S22), which is within $<10 \%$ of the theoretical limit. This certified $\sim 19.3 \mathrm{~mA} / \mathrm{cm}^{2}$ value is among the highest values for a single-pass device with 1.68-eV-band gap perovskites (Fig. 4H). In contrast with opaque devices with reflection from the rear electrode, single-junction devices required a thicker absorber to capture more photons near the band edge. With more photons absorbed by the top cell, we expect an enhanced upper limit of tandem efficiency.

Spectrally-weighted reflectances were calculated from the AM1.5 spectrum in the range of 350 to $900 \mathrm{~nm}$ (Fig. 4C). To investigate the effect of pyramid size on the reflection loss, we also selected different Si pyramid sizes by tailoring the texturing process. Although the pyramids in commercial $\mathrm{Si}$ are typically in the range of 2 to $7 \mu \mathrm{m}$, the benefit to reflection began to saturate when the pyramid size reached $2 \mu \mathrm{m}$ or less (see Fig. S23). This result is encouraging for perovskite-silicon tandems because it suggests that well-established solution-processing techniques can be united with textured silicon (Fig. S24).

We observed enhanced Jsc and FF in tandems (Figs. 4d, Figs. S24 and S25) in the case of fully textured c-Si bottom cells. EQE measurements highlight the advantage of switching from a polished front side to a double-sided textured architecture (Figs. 4, G and H). Reflections occurring in the flat design induced more current loss in the 330- to 1200-nm range. The introduction of a front texture reduced the overall reflectance, especially at wavelengths of 550 and $800 \mathrm{~nm}$. The $\mathrm{J}_{\mathrm{SC}}$ integrated from the EQE spectra is in excellent agreement with that derived from the 1-sun current density-voltage ( $\mathrm{J}-\mathrm{V})$ curve with enhanced $\mathrm{J}_{\mathrm{SC}}$ in both top cell and bottom cell on the double-side textured device.

Both differential EQE and absorption confirm the $~ 1.68-\mathrm{eV}$ perovskite band gap (Fig. S2). We observed narrower EQE overlap between the perovskite and Si cell. The textured device EQE maintained a plateau at 92 to $93 \%$ in this region. This result is distinct from previous work based on 400 - to 600 -nm-thick perovskite on flat devices in which the perovskite lost EQE at 
wavelengths of $550 \mathrm{~nm}$ and $800 \mathrm{~nm}$. This high EQE photocurrent, combined with enhanced charge extraction, resulted in high FF, leading to an improved device performance from $20.3 \%$ to $24.0 \%$ (Table S3). In particular, by further adding SLP treatment, we observed a distinct increase of FF from $72 \%$ to $77 \%$ (Fig. 4D). By reducing the recombination area and improved top contact design (Fig. S26), we achieved a Fraunhofer ISE certified stabilized (at MPP) PCE of $25.7 \%$ (Fig. 4, E and F, and Fig. S27).

The tandems reported herein show low hysteresis (Fig. 4E), high reproducibility (Fig. 4F and Fig. S28), and excellent operational stability under accelerated tests (Fig. 4, I to L). We monitored the stability of devices encapsulated with glass-butyl rubber. These devices were heated to $85^{\circ} \mathrm{C}$ for 400 hours in the dark at $\sim 40 \%$ relative humidity and found that devices retained their original performance (Fig. 4, I and J). We also monitored 400 hour operating stability of devices encapsulated using glass/POE (polyolefin encapsulant): here we used 1-sun-equivalent illumination (Fig. S29) at $40^{\circ} \mathrm{C}$ at $\sim 40$ to $50 \%$ relative humidity (Fig. 4, K and L). J-V curves in both scan directions ( $\mathrm{V}_{\mathrm{OC}}$ to $\mathrm{J}_{\mathrm{SC}}$ and $\mathrm{J}_{\mathrm{SC}}$ to $\mathrm{V}_{\mathrm{OC}}$ ) were measured at 10 -min intervals at $100 \mathrm{mV} / \mathrm{s}$ from $-0.1 \mathrm{~V}$ to $+1.9 \mathrm{~V}$ and vice versa. Between measurements, the tandem solar cell was held at the MPP voltage as determined by the most recent $\mathrm{J}-\mathrm{V}$ scan. Devices retained their original performance after $400 \mathrm{~h}$. In Fig. 4 , I to $\mathrm{K}$, the J-V curves of the tandem were reported prior to encapsulation, at the beginning of the test, and after $400 \mathrm{~h}$, and images are provided of devices at the end of the test. Overall, we attribute this good operating lifetime to the replacement of organic carrier-selective layers with $\mathrm{NiO}_{\mathrm{x}}$ inorganic materials, which prevent iodine reaction with smallmolecule organic HTLs (Table S4). 
A

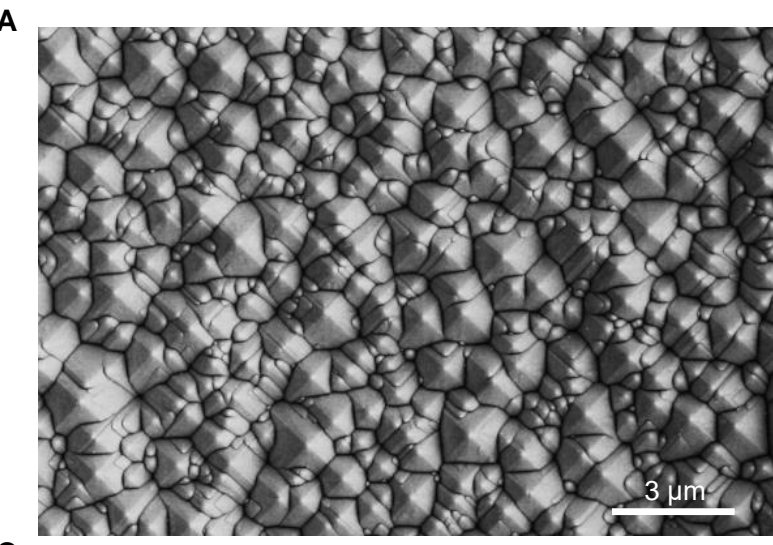

C
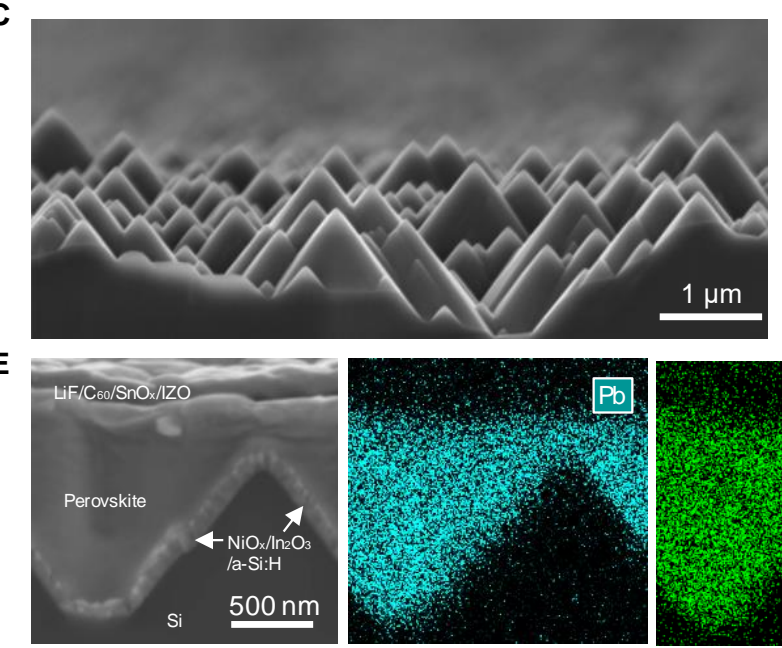

B

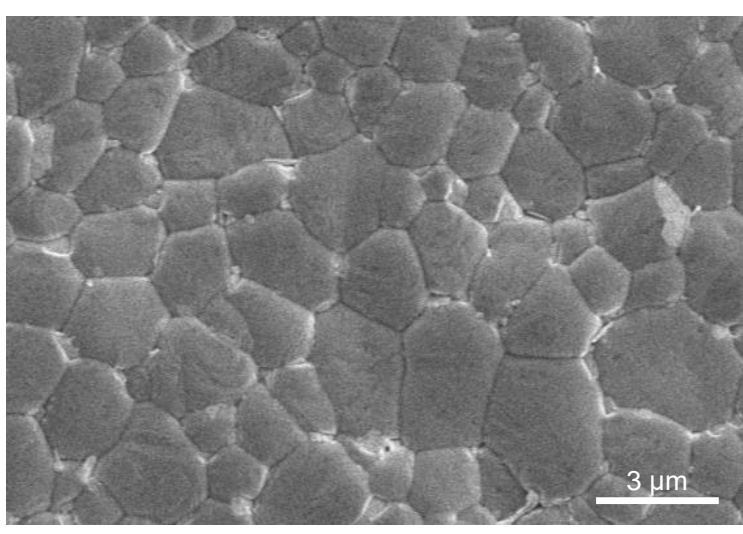

D
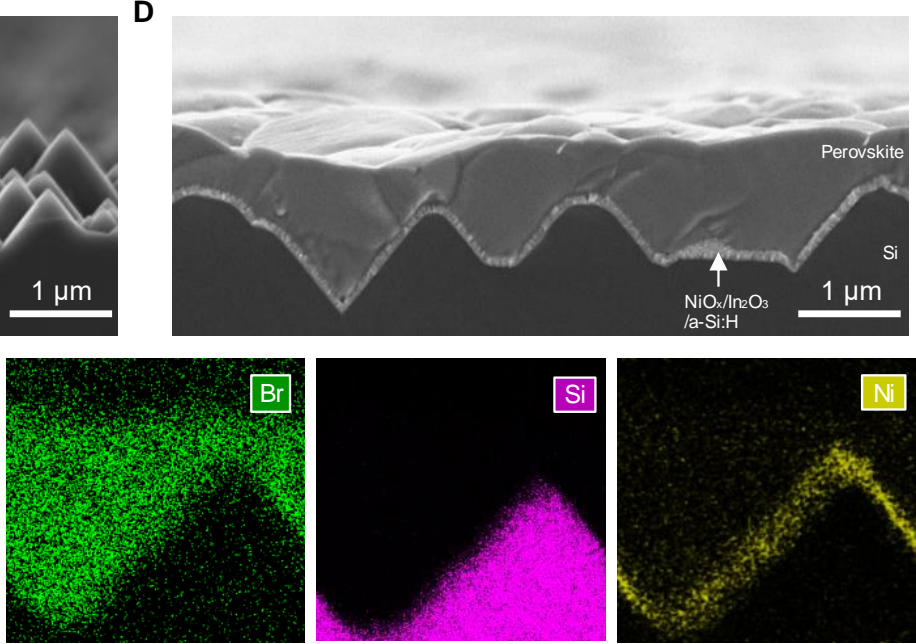

Fig. 1. Microstructure of solution-processed perovskite top cells on textured c-Si bottom cells. (A) SEM top-view images of textured c-Si surface with an average pyramid size of $2 \mu \mathrm{m}$, and (B) Corresponding substrates covered by solution-processed perovskite crystal. (C) SEM cross-section images of a textured c-Si with an average pyramid size of $2 \mu \mathrm{m}$, and (D) Corresponding substrates covered by solution-processed perovskite crystal. (E) SEM image with corresponding EDS maps denoting distribution of lead ( $\mathrm{Pb}$, aqua), bromide ( $\mathrm{Br}$, green), silicon (purple), and nickel (yellow) inside the solar cell. Lead and bromine distribution were confined to the perovskite layer. 

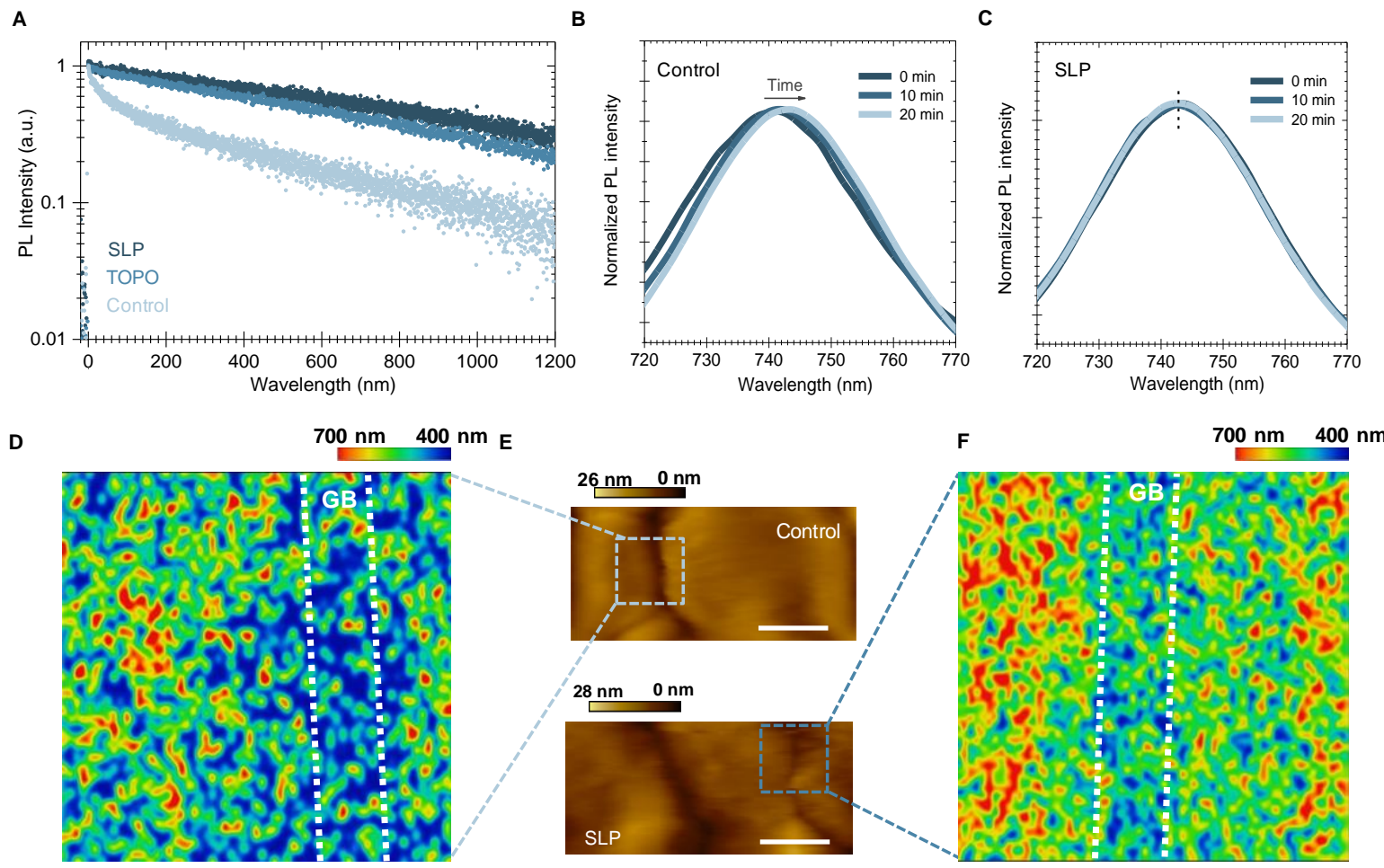

Fig. 2. Effects of SLP on diffusion length and phase segregation in micrometer-thick wide band gap perovskite. (A) Tr-PL spectrum of $1.68-\mathrm{eV}$-band gap thin films under different surface treatments. (B) Normalized PL spectra of control perovskite films after illuminating for 0,10 , and 20 min. (C) Normalized PL spectra of SLP-treated perovskite films after illuminating for 0, 10, and $20 \mathrm{~min}$. (D) Nanoscale-resolved mapping indicated by light blue square (100 $\mathrm{nm}$ by $100 \mathrm{~nm})$ of LD (diffusion length) of control perovskite. White dashed line shows the corresponding grainboundary area (E). Contact-mode AFM topography (upper) control sample and (lower) SLP perovskite (scale bar, $100 \mathrm{~nm}$ ). (F) Nanoscale-resolved mapping indicated by dark blue square (100 nm by $100 \mathrm{~nm}$ ) of LD (diffusion length) of SLP perovskite. White dashed line shows the corresponding grain-boundary area. 

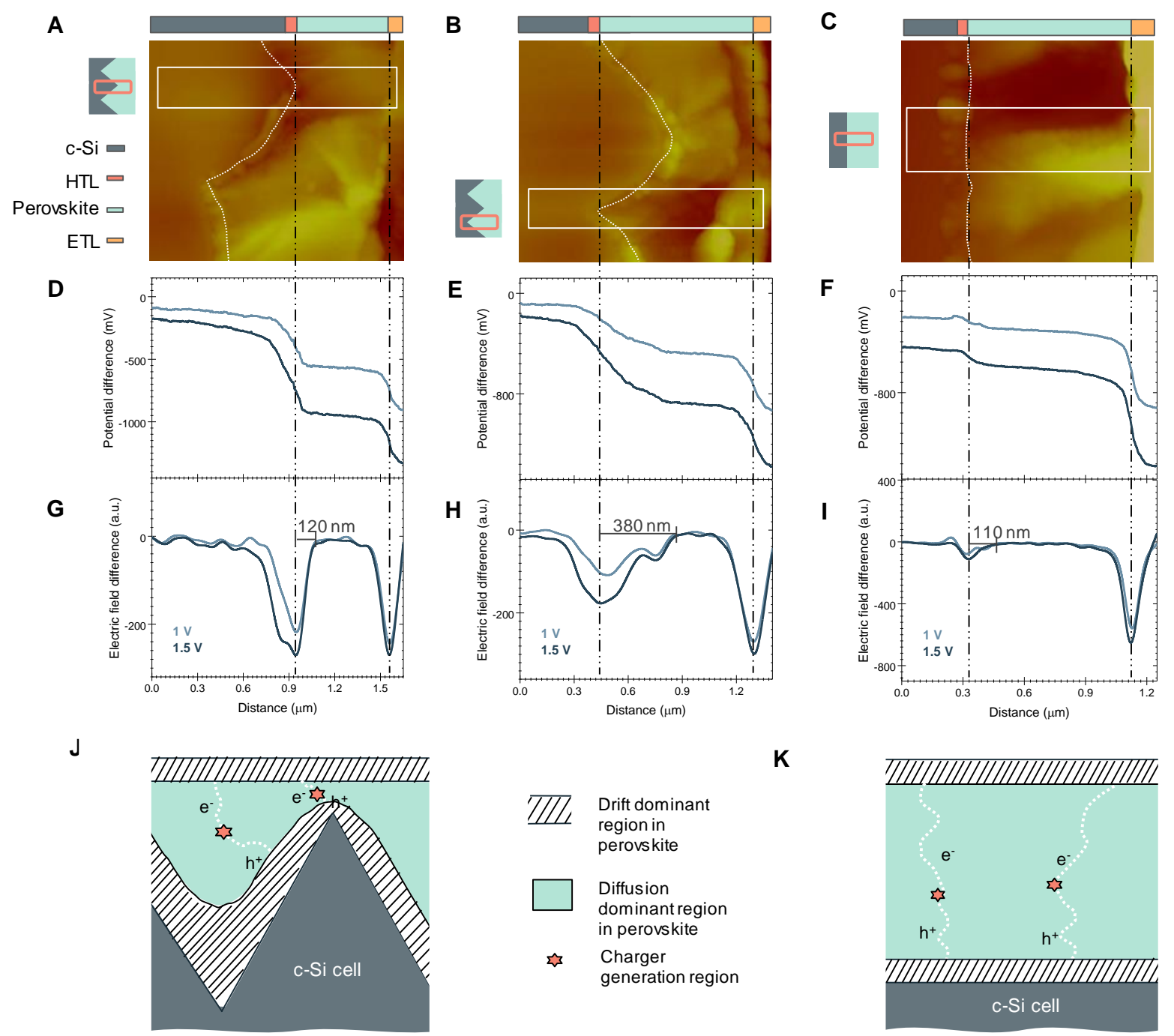

Fig. 3. Enhanced charge extraction in texture structured tandem cells. (A to C) AFM image of cross section of the peak of pyramid in textured and planar tandem with a schematic showing each layer across the top of Si bottom cell and the corresponding measured electric-potential zone. (D to F) Potential-difference profiling across the device under different bias voltages subtracted from the $0 \mathrm{~V}$ curve, and ( $\mathrm{G}$ to $\mathrm{I}$ ) Electric-field difference across the device, taken by the first derivative in (D to F). The corresponding KPFM results of flat cell. Schematics of electric-field distribution in textured $(\mathrm{J})$ and flat tandem devices $(\mathrm{K})$. 

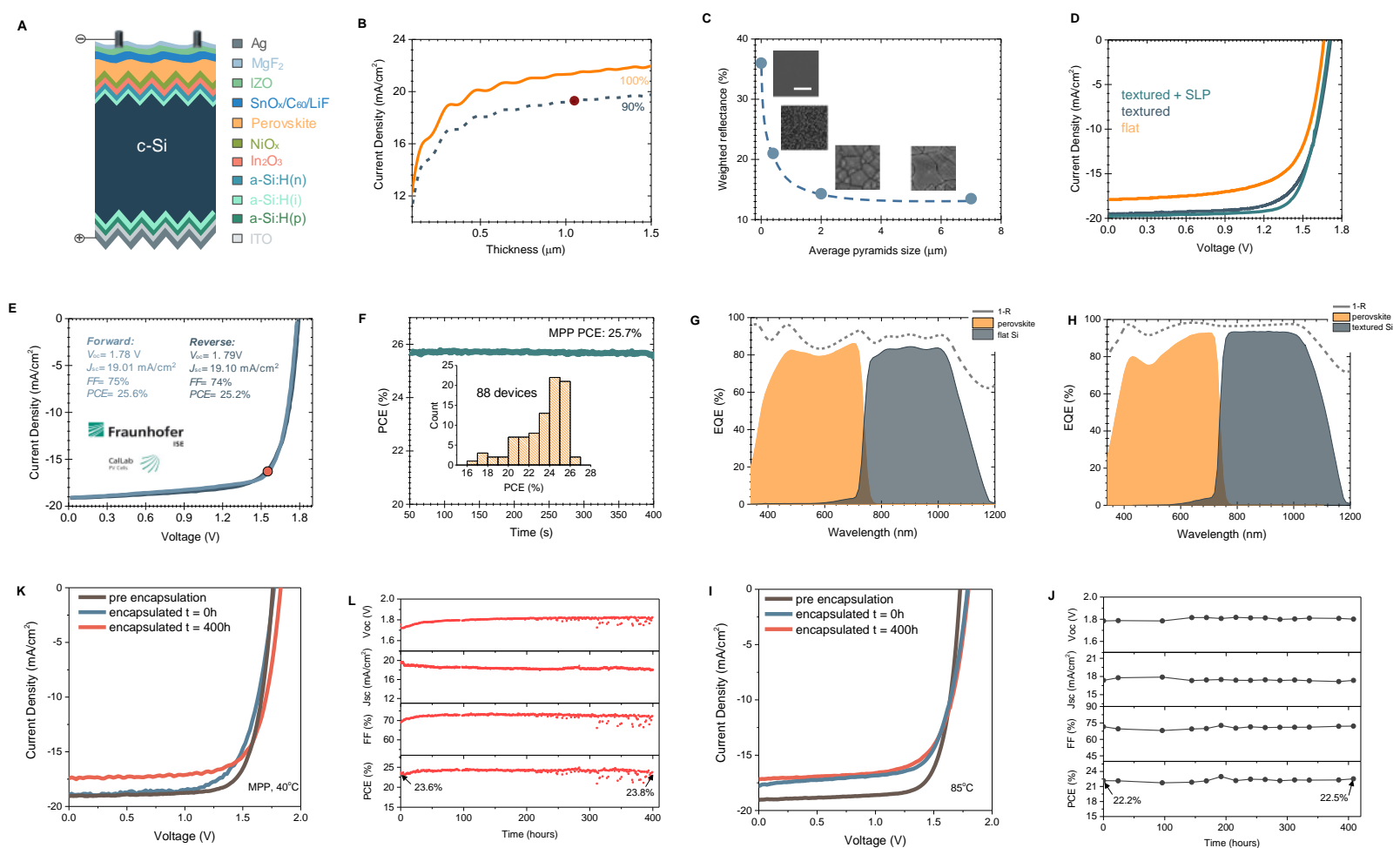

Fig. 4. Device characterization and stability of tandems. (A) Schematic of solution-processed perovskite/textured silicon tandem architecture. (B) Calculated JSC values of the perovskite cells as a function of perovskite layer thickness. Red spot represents the EQE-integrated JSC value in textured tandem top cell. (C) Measured weighted reflectance as a function of pyramid sizes of cSi. Texturing size refers to the pyramid base (scale bar, $2 \mu \mathrm{m}$ ). (D) J-V characteristics of flat and textured and SLP-treated textured tandems. J-V characteristics (E) of certified SLP-treated textured tandems. MPP tracking $(\mathrm{F})$ of certified SLP-treated textured tandems and PCE distributions of 88 devices. EQE of the flat $(\mathrm{G})$ and textured $(\mathrm{H})$ device (integrated current of 18.2 $\mathrm{mA} / \mathrm{cm}^{2}$ for top cell and $16.8 \mathrm{~mA} / \mathrm{cm}^{2}$ for bottom cell in flat device; $19.3 \mathrm{~mA} / \mathrm{cm}^{2}$ for top cell and $19.2 \mathrm{~mA} / \mathrm{cm}^{2}$ for bottom cell in Fraunhofer certified textured device). (I) $\mathrm{J}-\mathrm{V}$ curves of tandem devices before encapsulation (glass-butyl rubber), at the beginning, and the end of $85^{\circ} \mathrm{C}$ stability test; (J) J-V parameters measured over 400 hours stability test at $85^{\circ} \mathrm{C}$ (relative humidity $45 \%$ $50 \%$ ); (K) J-V curves of tandem devices before encapsulation (glass/POE), at the beginning and the end of the MPP stability tests; (L) J-V parameters measured > 400 hours of light-soaking, under MPP load at $40{ }^{\circ} \mathrm{C}$.

\section{References}

1. A. Kojima, K. Teshima, Y. Shirai, T. Miyasaka, Organometal Halide Perovskites as Visible-Light Sensitizers for Photovoltaic Cells. Journal of the American Chemical Society 131, 6050-6051 (2009).

2. NREL. (NREL, 2020), vol. 2020.

3. J. S. Manser, M. I. Saidaminov, J. A. Christians, O. M. Bakr, P. V. Kamat, Making and Breaking of Lead Halide Perovskites. Accounts of Chemical Research 49, 330-338 (2016). 
4. S. De Wolf et al., Organometallic Halide Perovskites: Sharp Optical Absorption Edge and Its Relation to Photovoltaic Performance. The Journal of Physical Chemistry Letters 5, 1035-1039 (2014).

5. D. P. McMeekin et al., A mixed-cation lead mixed-halide perovskite absorber for tandem solar cells. 351, 151-155 (2016).

6. Y. Deng et al., Surfactant-controlled ink drying enables high-speed deposition of perovskite films for efficient photovoltaic modules. Nature Energy 3, 560-566 (2018).

7. F. Sahli et al., Fully textured monolithic perovskite/silicon tandem solar cells with $25.2 \%$ power conversion efficiency. Nature Materials 17, 820-826 (2018).

8. M. Jošt et al., Textured interfaces in monolithic perovskite/silicon tandem solar cells: advanced light management for improved efficiency and energy yield. Energy \& Environmental Science 11, 3511-3523 (2018).

9. Q. Han et al., High-performance perovskite/Cu( $\mathrm{In}, \mathrm{Ga}) \mathrm{Se}_{2}$ monolithic tandem solar cells. 361, 904-908 (2018).

10. S. Essig et al., Raising the one-sun conversion efficiency of III-V/Si solar cells to $32.8 \%$ for two junctions and 35.9\% for three junctions. Nature Energy 2, 17144 (2017).

11. M. Saliba et al., Incorporation of rubidium cations into perovskite solar cells improves photovoltaic performance. 354, 206-209 (2016).

12. G. E. Eperon et al., Perovskite-perovskite tandem photovoltaics with optimized band gaps. 354, 861-865 (2016).

13. J. Tong et al., Carrier lifetimes of $>1 \mu$ in $\mathrm{Sn}-\mathrm{Pb}$ perovskites enable efficient all-perovskite tandem solar cells. 364, 475-479 (2019).

14. B. Chen et al., Grain Engineering for Perovskite/Silicon Monolithic Tandem Solar Cells with Efficiency of 25.4\%. Joule 3, 177-190 (2019).

15. K. A. Bush et al., 23.6\%-efficient monolithic perovskite/silicon tandem solar cells with improved stability. Nature Energy 2, 17009 (2017).

16. H. Shen et al., In situ recombination junction between $\mathrm{p}-\mathrm{Si}$ and $\mathrm{TiO}_{2}$ enables highefficiency monolithic perovskite/Si tandem cells. 4, eaau9711 (2018).

17. F. Fu et al., High-efficiency inverted semi-transparent planar perovskite solar cells in substrate configuration. Nature Energy 2, 16190 (2016).

18. J. Zheng et al., Large area efficient interface layer free monolithic perovskite/homojunction-silicon tandem solar cell with over $20 \%$ efficiency. Energy \& Environmental Science 11, 2432-2443 (2018).

19. W. S. Yang et al., Iodide management in formamidinium-lead-halide-based perovskite layers for efficient solar cells. 356, 1376-1379 (2017).

20. Q. Jiang et al., Surface passivation of perovskite film for efficient solar cells. Nature Photonics 13, 460-466 (2019).

21. L. Gil-Escrig et al., Vacuum Deposited Triple-Cation Mixed-Halide Perovskite Solar Cells. 8, 1703506 (2018).

22. E. Aydin, M. De Bastiani, S. De Wolf, Defect and Contact Passivation for Perovskite Solar Cells. 31, 1900428 (2019).

23. I. L. Braly et al., Hybrid perovskite films approaching the radiative limit with over $90 \%$ photoluminescence quantum efficiency. Nature Photonics 12, 355-361 (2018).

24. D. W. de Quilettes et al., Impact of microstructure on local carrier lifetime in perovskite solar cells. 348, 683-686 (2015). 
25. R. A. Belisle et al., Impact of Surfaces on Photoinduced Halide Segregation in MixedHalide Perovskites. ACS Energy Letters 3, 2694-2700 (2018).

26. E. Aydin et al., Room-Temperature-Sputtered Nanocrystalline Nickel Oxide as Hole

Transport Layer for $\mathrm{p}-\mathrm{i}-\mathrm{n}$ Perovskite Solar Cells. ACS Applied Energy Materials 1, 6227-6233 (2018).

27. P. P. Maharjan et al., Photovoltaic devices and characterization of a dodecyloxybenzothiadiazole-based copolymer. Physical Chemistry Chemical Physics 15, 68566863 (2013).

28. Y. Xie et al., Electrolyte Effects on Electron Transport and Recombination at $\mathrm{ZnO}$ Nanorods for Dye-Sensitized Solar Cells. The Journal of Physical Chemistry C 114, 17880-17888 (2010).

29. A. B. Martinson et al., Electron transport in dye-sensitized solar cells based on $\mathrm{ZnO}$ nanotubes: evidence for highly efficient charge collection and exceptionally rapid dynamics. The Journal of Physical Chemistry A 113, 4015-4021 (2009).

30. K. A. Bush et al., 23.6\%-efficient monolithic perovskite/silicon tandem solar cells with improved stability. Nature Energy, (2017).

31. L. Mazzarella et al., Infrared Light Management Using a Nanocrystalline Silicon Oxide Interlayer in Monolithic Perovskite/Silicon Heterojunction Tandem Solar Cells with Efficiency above 25\%. Advanced Energy Materials 0, 1803241 (2019).

32. E. Köhnen et al., Highly Efficient Monolithic Perovskite Silicon Tandem Solar Cells: Analyzing the Influence of Current Mismatch on Device Performance. Sustainable Energy \& Fuels, (2019).

33. F. Sahli et al., Fully textured monolithic perovskite/silicon tandem solar cells with $25.2 \%$ power conversion efficiency. Nature Materials, (2018).

\section{ACKNOWLEDGMENTS}

The manuscript was improved by the insightful reviews provided by anonymous reviewers. Funding: This publication is based upon work supported by the King Abdullah University of Science and Technology (KAUST) Office of Sponsored Research (OSR) under Award No. OSR2018-CPF-3669.02 and in part on work supported by the US Department of the Navy, Office of Naval Research (Grant Award No.: N00014-17-1-2524). This work was authored in part by the National Renewable Energy Laboratory, operated by Alliance for Sustainable Energy, LLC, for the U.S. Department of Energy (DOE) under Contract No. DE-AC36-08GO28308. Funding provided by applicable Department of Energy office and program office, e.g., U.S. Department of Energy Office of Energy Efficiency and Renewable Energy Solar Energy Technologies Office. The views expressed in the article do not necessarily represent the views of the DOE or the U.S. Government. The U.S. Government retains and the publisher, by accepting the article for publication, acknowledges that the U.S. Government retains a nonexclusive, paid-up, irrevocable, worldwide license to publish or reproduce the published form of this work, or allow others to do so, for U.S. Government purposes. This work has been partially supported by NSF MRI (1428992), U.S. - Egypt Science and Technology (S\&T) Joint Fund, and EDA University Center Program (ED18DEN3030025). This work is derived from the Subject Data supported in whole or part by NAS and USAID, and any opinions, findings, conclusions, or recommendations expressed in the paper are those of the authors alone, and do not necessarily reflect the views of USAID or NAS. 
Authors contributions: Y.H., E.A., and M.D.B. conceived the idea and designed the experiments. Y.H., E.A., and M.D.B. fabricated the devices and conducted the JV and EQE characterizations. C.X. conducted KPFM study, C.X. and K.Z. analyzed and discussed the KPFM results, C.X. wrote the KPFM portion of the manuscript. F. H. I. developed the perovskites holes extraction layer. J. T. developed the setup for stability and performed the mpp test. M.D.B. and R. J. developed the silicon texturing. M.D.B. and A.J.M. realized the statistics of the devices, M.D.B, T.A., E.V.K. and R.J. developed the silicon bottom cell. D.B. supervised the stability test. B.C. contributed to SEM measurements. D.J.X, and H.C. contributed to perovskite fabrication and passivation analysis. M.Y., Z.H., and Y.D. contributed to PL and TR measurements. A.J. performed GIWAXS measurements. S. W. B. contributed to FDTD simulations. Q.Q., B.B., and A.H.C. developed and implement the mapping of TPV/TPC, analyzed the results, and wrote the TPV/TPC mapping portion of the manuscript. D.B., M.I.S., K.Z., and E.H.S. provided advices. Y.H., E.A., M.D.B., C.X., S.D.W., and E.H.S. composed the manuscript. All authors discussed the results and commented on the manuscript. Competing interests: None declared. Data and materials availability: All data needed to evaluate the conclusions in the paper are present in the paper or the supplementary materials 